\title{
Dark matter within the minimal flavour violation ansatz
}

\author{
Laura Lopez-Honorez ${ }^{a}$, Luca Merlo ${ }^{\mathrm{b}, \mathrm{c}, *}$ \\ a Theoretische Natuurkunde, Vrije Universiteit Brussel and The International Solvay Institutes Pleinlaan 2, B-1050 Brussels, Belgium \\ ${ }^{\mathrm{b}}$ Instituto de Física Teórica UAM/CSIC and Departamento de Física Teórica, Universidad Autónoma de Madrid, Cantoblanco, 28049 Madrid, Spain \\ c CERN, Department of Physics, Theory Division, $\mathrm{CH}-1211$ Geneva 23, Switzerland
}

\section{A R T I C L E I N F O}

\section{Article history:}

Received 10 March 2013

Accepted 8 April 2013

Available online 9 April 2013

Editor: G.F. Giudice

\begin{abstract}
A B S T R A C T
Minimal Flavour Violation hypothesis can provide an attractive framework for Dark Matter (DM). We consider scalar DM candidates carrying flavour quantum numbers and whose representation under the flavour group guarantees DM stability. They interact with the Standard Model fields through Higgs portal at renormalisable level and also to quarks through dimension-6 operators. We provide a systematic analysis of the viable parameter space for the DM fields, which are triplet of the flavour group, considering several DM-quark interactions. In this framework, we analyse in which cases the viable parameter space differs from Higgs portal models thanks to the underlying flavour structure. In contrast to minimal Higgs portal scenarios, we find that light DM in the GeV mass range as well as heavier candidates above Higgs resonance could be allowed by colliders, direct and indirect DM detection searches as well as flavour constraints. The large mass regime above $m_{t}$ could even be beyond the reach of future experiments such as Xenon 1T.
\end{abstract}

(C) 2013 Elsevier B.V. All rights reserved.

\section{Introduction}

The determination of the nature of DM is one of the main challenges of cosmology and particle physics community today. The Standard Model (SM) of particle physics, despite its great successes along with the likely recent unveiling of its last building block at LHC [1,2], fails in providing a satisfactory DM candidate. In order to characterise the properties of the DM particle, we are forced to go beyond the SM. As of today, no clear scenario for New Physics (NP) is emerging. Concerning DM, we still ignore its intrinsic properties, how it couples to the SM particles and if the dark sector is made of one or several species.

Within the SM, the SM scalar or Brout-Englert-Higgs ("Higgs" for short) boson enjoys a special status since it can allow for a direct coupling to the dark sector at renormalisable level. It is already well known that the so-called "Higgs portal" provides a quite simple and attractive framework for DM phenomenology [3-20]. Interestingly, Higgs searches offer complementary bounds to direct and indirect DM detection searches on the viable parameter space of such DM models, especially in the low mass region [21-23,16, 24-28].

\footnotetext{
* Corresponding author at: Instituto de Física Teórica UAM/CSIC and Departamento de Física Teórica, Universidad Autónoma de Madrid, Cantoblanco, 28049 Madrid, Spain.

E-mail addresses: llopezho@vub.ac.be (L. Lopez-Honorez), luca.merlo@uam.es (L. Merlo).

The DM stability requirement is usually ensured by imposing by hand a discrete $Z_{2}$ symmetry, under which the DM candidate is odd while the SM fields are even. Several works have however been investigating which models of NP could provide a more fundamental origin for its stability, see e.g. Ref. [29] for a short review. A particular remark should be make on models where more sophisticated global discrete symmetries [30-44] and horizontal gauge symmetries [45-48] have been adopted to deal with the DM phenomenology.

Recently, Ref. [49] sketched the typical features of DM candidates coupling to quarks in a way that is consistent with the Minimal Flavour Violation (MFV) ansatz [50,51]. Interestingly, the MFV, originally motivated to suppress dangerous flavour changing neutral current (FCNC) processes in NP contexts, can have a dual purpose guaranteeing the stability of the DM.

MFV is a working context that has been codified in Refs. [5259] as a general framework built upon the flavour symmetry of the kinetic terms of the SM Lagrangian. Focusing on the quark sector only, the latter presents a global flavour symmetry whose non-Abelian part is given by

$G_{f}=S U(3)_{Q_{L}} \times S U(3)_{U_{R}} \times S U(3)_{D_{R}}$

$G_{f}$ is only broken by the Yukawa interactions, unless the Yukawa matrices are promoted to be auxiliary fields, called spurions, transforming non-trivially under $G_{f}$. Without entering into details of a specific NP scenario, generic effects of flavour and CP violation can be described by means of an effective Lagrangian. If all the 
effective operators of this Lagrangian are constructed by means of the SM fields and the Yukawa spurions, all the flavour bounds are satisfied with a NP scale of few TeV [52-54,60,61,55,62,63,58,6467].

A stable DM candidate can arise within the MFV context when the DM is assumed to carry flavour quantum numbers (see also Refs. [68-70]) and the DM field representation under $G_{f}$ is chosen in order to prevent DM decay into SM particles. Following the approach of Ref. [49], we assume that the DM fields are neutral under the SM gauge group, but transforms under $G_{f}$. For simplicity, we consider new scalar degrees of freedom as DM candidates with the lowest representation under $G_{f}$ that guarantees their stability. By construction, the DM flavour-multiplets do not couple at the renormalisable level to quarks. Their interactions with SM particles result from the interplay between Higgs portal interactions and effective dimensions-six operators coupling DM to quarks. Notice that Ref. [49] proposed the rules for stable flavoured DM. In that Letter, the DM analysis focused on scalar DM interacting through one particular $d=6$ operator and with a fixed hierarchy in the DM components. The authors provided a survey of the typical bounds that have to be taken into account from DM and flavour physics. This study missed though several new features in the DM viable parameter space that distinguish the DM within the MFV context compared to the standard Higgs portal DM scenarios.

In this Letter, our purpose is to perform a systematic analysis of the viable parameter space of such DM context and to study how it differs from the simple singlet scalar DM interacting via Higgs portal. In particular, we obtain new features in the few $\mathrm{GeV}$ mass range, thanks to DM coannihilation induced by flavour symmetry, as well as larger viable parameter space for the DM mass range above the top mass $m_{D M}>m_{t}$, when an extra interaction to $u$-type quarks is considered.

In the following, we first briefly summarise in Section 2 the MFV context and its extension involving a potential DM candidate. In Section 3, we review the general form of effective $d=6$ operators providing DM-quark interactions compatible with the flavour symmetry. In Section 4, we study the parameter space of this DM scenario considering bounds from direct and indirect DM and collider searches and flavour constraints, and conclude in Section 5.

\section{The MFV-DM context}

Deviations from the SM predictions induced by NP with generic flavour structure are already severely constrained by the increasing accuracy in the determination of the CKM matrix elements, the measurement of a large number of FCNC processes and of $\mathrm{CP}$ asymmetries. In a general context, the scale of NP responsible for the flavour interactions should be above hundreds or thousands $\mathrm{TeV}$ [71]. On the other hand, under the MFV hypothesis which prescribes that all the sources of flavour and CP violation in any NP scenario are the same as in the SM, the scale of NP responsible for the flavour interactions is lowered down to few TeV.

This is technically implemented by constructing effective operators describing flavour and $\mathrm{CP}$ violation by means of quarks and Yukawa spurions, that transform under the flavour group $G_{f}$ in Eq. (1). The $S U(2)_{L}$ doublet $Q_{L}$ and singlets $u_{R}$ and $d_{R}$ transform according to

$Q_{L} \sim(\mathbf{3}, \mathbf{1}, \mathbf{1}), \quad u_{R} \sim(\mathbf{1}, \mathbf{3}, \mathbf{1}), \quad d_{R} \sim(\mathbf{1}, \mathbf{1}, \mathbf{3})$,

while the Yukawa spurions transform as

$Y_{u} \sim(\mathbf{3}, \overline{\mathbf{3}}, \mathbf{1}), \quad Y_{d} \sim(\mathbf{3}, \mathbf{1}, \overline{\mathbf{3}})$,

ensuring the invariance under $G_{f}$ of the Yukawa Lagrangian,
$\mathscr{L}_{Y}=-\bar{Q}_{L} \tilde{H} Y_{u} u_{R}-\bar{Q}_{L} H Y_{d} d_{R}+$ h.c.

Quark masses and mixings are then correctly reproduced (but not predicted [57]) once these spurion fields get background values as

$Y_{u}=\mathbf{y}_{u}, \quad Y_{d}=V \mathbf{y}_{d}$,

where $\mathbf{y}_{u, d}$ are diagonal matrices whose elements are the Yukawa eigenvalues, and $V$ is the CKM matrix.

Stable DM candidates can be found within the MFV framework looking for the representations of $G_{f}$ that forbid the construction of operators inducing the DM decay into SM degrees of freedom. It has been shown [49] that the lowest representation under $G_{f}$ providing a stable DM candidate is a triplet under one of the $S U(3)_{i}$ composing $G_{f}$. The DM stability is then insured for any Lorentz representation of the DM candidate. For definiteness, we consider scalar DM $S$, neutral under the SM gauge group, and focus on the representation

\section{$S \sim(3, \mathbf{1}, \mathbf{1})$}

under $G_{f}$. Let us emphasise that our conclusions on the DM phenomenology should apply to any scalar triplet under one $S U(3)_{i}$ term of $G_{f}$, after a slight modification of the dimension- 6 operators that drive its interactions to the quarks (see Section 3).

The low-energy effective Lagrangian describing our setup is given by

$\mathscr{L}=\mathscr{L}_{S M}+\partial^{\mu} S^{\dagger} \partial_{\mu} S-V(S, H)+\mathscr{L}_{\text {eff }}^{f}+\mathscr{L}_{\text {eff }}^{D M}$,

where $\mathscr{L}_{S M}$ is the SM Lagrangian, $V$ is the scalar potential involving the DM field $S$ and the Higgs doublet $H . \mathscr{L}_{\text {eff }}^{f}$ could contain $d=6$ pure-flavour operators, described in e.g. Ref. [52], that are suppressed by $\Lambda_{f}^{2}$, while $\mathscr{L}_{\text {eff }}^{D M}$ contains $d=6$ DM-flavour operators suppressed by $\Lambda_{D M}^{2} . \Lambda_{f}$ and $\Lambda_{D M}$ are the characteristic mass scales of the messengers of the pure-flavour and DM-flavour interactions, respectively, and can a priori be distinct. While $\Lambda_{f}$ should be larger than a few $\mathrm{TeV}$ [52] to satisfy all flavour constraints, lower values of $\Lambda_{D M}$ are still allowed. In practice, we do not explicitly include the contribution of $\mathscr{L}_{\text {eff }}^{f}$ for the numerical analysis in Section 4.3 , as $\mathscr{L}_{\text {eff }}^{f}$ is not expected to affect the DM phenomenology given that in our analysis $\Lambda_{f}$ is always larger than $\Lambda_{D M}$.

The SM scalar potential get modified at the renormalisable level by the introduction of new scalar degrees of freedom. As already described in Ref. [49], in the MFV context, the new contributions to the scalar potential $V$ read

$$
\begin{aligned}
V \supset & m_{S}^{2} S_{i}^{*}\left(a \mathbf{1}_{i j}+b\left(Y_{u} Y_{u}^{\dagger}\right)_{i j}+\cdots\right) S_{j} \\
& +\lambda S_{i}^{*}\left(a^{\prime} \mathbf{1}_{i j}+b^{\prime}\left(Y_{u} Y_{u}^{\dagger}\right)_{i j}+\cdots\right) S_{j} H^{\dagger} H,
\end{aligned}
$$

where $i, j$ are flavour indices, $a, b, a^{\prime}, b^{\prime}$ are dimensionless $\mathcal{O}(1)$ parameters and the ellipsis denotes further negligible ${ }^{1} Y_{d}$ spurion insertions. After flavour and electroweak symmetry breaking (EWSB), $\langle H\rangle=\mathrm{v} / \sqrt{2}$ with $\mathrm{v}=246 \mathrm{GeV}$, the $S$ mass-squared matrix is diagonal and given by

$\mathscr{L}_{m} \supset-S_{i}^{*}\left[m_{A}^{2}+m_{B}^{2} \mathbf{y}_{u_{i}}^{2}\right] S_{i}$,

where we have defined

$m_{A}^{2}=m_{S}^{2} a+\frac{1}{2} \lambda v^{2} a^{\prime}, \quad m_{B}^{2}=m_{S}^{2} b+\frac{1}{2} \lambda v^{2} b^{\prime}$.

\footnotetext{
1 Here we follow the common practice considering all interactions up to the first power in $Y_{u} Y_{u}^{\dagger}$. See e.g. Ref. [61] for an analysis studying the impact of resuming over the Yukawa coupling expansion in the context of MFV in the absence of DM.
} 
Of phenomenological importance are the trilinear couplings of the DM particles to the physical Higgs $h$, which in the mass eigenbasis read:

$\mathscr{L} \supset-\frac{1}{2} \lambda v h S_{i}^{*}\left(a^{\prime}+b^{\prime} \mathbf{y}_{u_{i}}^{2}\right) S_{i} \equiv-\frac{1}{2} \lambda_{i} v h S_{i}^{*} S_{i}$,

where $\lambda_{i} \equiv \lambda\left(a^{\prime}+b^{\prime} \mathbf{y}_{u_{i}}^{2}\right)$.

\section{The DM-flavour operators}

$S$ transforming as a triplet of $G_{f}$ as in Eq. (6) prevents the construction of any operator containing a single DM field and quarks. A coupling between pairs of DM multiplets and quarks is still allowed through non-renormalisable interactions suppressed by $\Lambda_{D M}^{2}$. When the flavour symmetry $G_{f}$ and the EW symmetry are unbroken, one can consider the following $d=6$ operators:

$\mathscr{L}_{\text {eff }}^{D M}=\frac{1}{\Lambda_{D M}^{2}} \sum_{\alpha=1}^{5} c_{i j k \ell}^{\alpha}\left(\mathcal{O}_{\alpha}\right)_{i j k \ell}$,

with

$$
\begin{aligned}
& \left(\mathcal{O}_{1}\right)_{i j k \ell}=\left(\bar{Q}_{L_{i}} \gamma^{\mu} Q_{L_{j}}\right)\left(S_{k}^{*} \overleftrightarrow{\partial_{\mu}} S_{\ell}\right), \\
& \left(\mathcal{O}_{2}\right)_{i j k \ell}=\left(\bar{u}_{R_{i}} \gamma^{\mu} u_{R_{j}}\right)\left(S_{k}^{*} \overleftrightarrow{\partial_{\mu}} S_{\ell}\right), \\
& \left(\mathcal{O}_{3}\right)_{i j k \ell}=\left(\bar{d}_{R_{i}} \gamma^{\mu} d_{R_{j}}\right)\left(S_{k}^{*} \overleftrightarrow{\partial_{\mu}} S_{\ell}\right), \\
& \left(\mathcal{O}_{4}\right)_{i j k \ell}=\left(\bar{Q}_{L_{i}} u_{R_{j}}\right)\left(S_{k}^{*} S_{\ell}\right) \tilde{H}+\text { h.c., } \\
& \left(\mathcal{O}_{5}\right)_{i j k \ell}=\left(\bar{Q}_{L_{i}} d_{R_{j}}\right)\left(S_{k}^{*} S_{\ell}\right) H+\text { h.c., }
\end{aligned}
$$

where $i, j, k, \ell$ are flavour indices (see also Refs. [72,73]). The operators above correspond to an effective theory description of the NP sector giving rise to an additional sources of quark-DM coupling besides the Higgs portal interactions. The coefficients $c_{i j k \ell}^{\alpha}$ take into account all possible flavour contractions and read as

$$
\begin{aligned}
c_{i j k \ell}^{1}= & c_{1}^{1} \mathbf{1}_{i j} \mathbf{1}_{k \ell}+c_{2}^{1} \mathbf{1}_{i \ell} \mathbf{1}_{k j}+c_{3}^{1}\left(Y_{u} Y_{u}^{\dagger}\right)_{i j} \mathbf{1}_{k \ell} \\
& +c_{4}^{1} \mathbf{1}_{i j}\left(Y_{u} Y_{u}^{\dagger}\right)_{k \ell}+c_{5}^{1}\left(Y_{u} Y_{u}^{\dagger}\right)_{i \ell} \mathbf{1}_{k j}+\cdots, \\
c_{i j k \ell}^{2}= & c_{1}^{2} \mathbf{1}_{i j} \mathbf{1}_{k \ell}+c_{2}^{2}\left(Y_{u}^{\dagger} Y_{u}\right)_{i j} \mathbf{1}_{k \ell} \\
& +c_{3}^{2} \mathbf{1}_{i j}\left(Y_{u} Y_{u}^{\dagger}\right)_{k \ell}+c_{4}^{2}\left(Y_{u}^{\dagger}\right)_{i \ell}\left(Y_{u}\right)_{k j}+\cdots, \\
c_{i j k \ell}^{3}= & c_{1}^{3} \mathbf{1}_{i j} \mathbf{1}_{k \ell}+c_{2}^{3}\left(Y_{d}^{\dagger} Y_{d}\right)_{i j} \mathbf{1}_{k \ell} \\
& +c_{3}^{3} \mathbf{1}_{i j}\left(Y_{u} Y_{u}^{\dagger}\right)_{k \ell}+c_{4}^{3}\left(Y_{d}^{\dagger}\right)_{i \ell}\left(Y_{d}\right)_{k j}+\cdots, \\
c_{i j k \ell}^{4}= & c_{1}^{4}\left(Y_{u}\right)_{i j} \mathbf{1}_{k \ell}+c_{2}^{4} \mathbf{1}_{i \ell}\left(Y_{u}\right)_{k j} \\
& +c_{3}^{4}\left(Y_{u}\right)_{i j}\left(Y_{u} Y_{u}^{\dagger}\right)_{k \ell}+c_{4}^{4}\left(Y_{u} Y_{u}^{\dagger}\right)_{i \ell}\left(Y_{u}\right)_{k j}+\cdots, \\
c_{i j k \ell}^{5}= & c_{1}^{5}\left(Y_{d}\right)_{i j} \mathbf{1}_{k \ell}+c_{2}^{5} \mathbf{1}_{i \ell}\left(Y_{d}\right)_{k j} \\
& +c_{3}^{5}\left(Y_{d}\right)_{i j}\left(Y_{u} Y_{u}^{\dagger}\right)_{k \ell}+c_{4}^{5}\left(Y_{u} Y_{u}^{\dagger}\right)_{i \ell}\left(Y_{d}\right)_{k j}+\cdots,
\end{aligned}
$$

where we have considered all possible terms up to first powers in $Y_{u} Y_{u}^{\dagger}$ and the dots refer to negligible contractions associated to $Y_{d}$ insertions. In the following, we use real coefficients $c_{a}^{\alpha}$, according to the MFV ansatz under which all the sources of flavour and CP violation should be associated to the Yukawas only.

The scale $\Lambda_{D M}$ in Eq. (12) corresponds to a function of couplings and mediator masses whose exact combination depends on the UV completion of the effective theory under study. In principle, different operators or flavour contractions can depend on different type of messengers. $\mathcal{O}_{1,2,3}$ would for instance be the result of a vector boson exchange, while $\mathcal{O}_{4,5}$ would be due to the exchange of scalar or fermionic mediators. Here we assume that the structure of Eqs. (12)-(22) captures the DM phenomenology as long as the DM mass is lighter than the particles mediating the interactions.

Also notice that when the energy scale involved in the physical processes is below the EWSB scale, both the gauge $S U(2)_{L} \times U(1)_{Y}$ and the flavour $G_{f}$ symmetry descriptions used in the definition of Eqs. (13)-(17) break down. One could then wonder for the appearance of new kind of operators, especially for those inducing DM decay, but this is not the case: the effective theory at low energy must match the theory at higher energies and therefore no new gauge or flavour couplings appear.

\section{The viable parameter space}

In this section, we study the viable parameter space for DM analysing the impact of each dimension- 6 operator $\mathcal{O}_{1, \ldots, 5}$ on DM phenomenology.

\subsection{Constraints from DM and flavour physics}

Let us first summarise the constraints that have been imposed in each scenarios. We consider that $\lambda_{i}<\pi$ in order to preserve a perturbative regime. We take $\lambda_{i}>-\sqrt{\pi \lambda_{h}}$, where $\lambda_{h}$ is the Higgs self-coupling, to ensure that the scalar potential is bounded from below, assuming that the DM scalar self-couplings are up to $\sim \mathcal{O}(\pi)$. We also impose that $\Lambda_{D M}>m_{S_{1}}, m_{S_{2}}, m_{S_{3}}$ to avoid breaking down of the effective field theory description. In addition, from DM and flavour physics, we have to take into account the following points:

1. Relic abundance $\Omega_{D M}$ : The total DM abundance deduced from cosmological observations constrains the thermally averaged effective annihilation cross-section $\langle\sigma v\rangle$. In the simplest cases [74], for the Taylor expanded annihilation cross section times the centre of mass velocity $\sigma v=a+b v^{2}$, the Boltzmann equations for the evolution of DM number density gives rise to the relation

$$
\Omega_{D M} h^{2}=\frac{1.7 \times 10^{9} x_{f} \mathrm{GeV}^{-1}}{\sqrt{g_{*}}\left(a+3 b / x_{f}\right) m_{p l}}
$$

where $h$ is the Hubble parameter, ${ }^{2} x_{f}$ is the ratio between the DM mass and its freeze out temperature, $g_{*}$ is the number of relativistic degrees of freedom and $m_{p l}=1.22 \times 10^{19} \mathrm{GeV}$ is the Planck mass. The constraint $\Omega_{D M} h^{2} \sim 0.1$ [75] translates then into $\langle\sigma v\rangle \sim 3 \times 10^{-26} \mathrm{~cm}^{3} / \mathrm{s}$ or equivalently $\langle\sigma v\rangle \sim$ $3 \times 10^{-9} \mathrm{GeV}^{-2}$. In our numerical analysis of Section 4.2 , we use the code MicromeGAs [76,77] that integrates more accurately the set of Boltzmann equations and we impose $0.09<\Omega_{D M} h^{2}<0.13$.

2. Direct and indirect detection: Direct detection searches are among the best test of Weakly Interacting Massive Particle (WIMP) DM scenarios. This is especially the case of spin independent DM-proton scattering and for masses around $50 \mathrm{GeV}$. The associated cross-section $\sigma_{D M p}$ should be below the bounds of experiments such as PICASSO [78] and Xenon 100 [79] for $m_{D M}$ in the GeV-TeV range. Notice that such experiments have an energy threshold in the few $\mathrm{GeV}$ range and PICASSO's threshold is among the lowest ones.

Indirect detection searches are also digging into the viable WIMP mass range. The annihilation cross-section times centre

\footnotetext{
2 Here, we keep the notation $h$ for the Hubble parameter as it is widely used in the literature. It should not be confused with the physical Higgs field
} 
of mass velocity $\sigma v$, can be tested by FERMI experiment [80]. The Cosmic Microwave Background (CMB) observation experiments such as WMAP [75], SPT [81] and Planck [82] (in the very near future) provide complementary constraints on $\sigma v$. Indeed, the energy released into the Inter Galactic Medium (IGM) by DM annihilation can alter the thermal history of the Universe, leading to observable changes in CMB observables, see $[83-86]$ and $[87,88]$ for the latest analysis.

3. Colliders: Assuming that the particle resonance with a mass of about $125 \mathrm{GeV}$ observed by the ATLAS and CMS Collaborations at the LHC $[1,2]$ corresponds to the Higgs boson, one can constrain the invisible branching ratio to be $\operatorname{Br}(h \rightarrow$ inv $)<0.15$ at $2 \sigma$ level [89]. Latest constraints from monojet events observed by the CMS [90] and ATLAS [91] Collaborations can give rise to stringent bounds on $\sigma_{D M} p$ complementary to direct detection searches for WIMP masses in the $\mathrm{GeV}$ range [92,93]. Let us emphasise that the limits were derived for fermionic DM and in the case of scalar DM these bounds are not always that constraining. In the following, we use the results presented in Ref. [94].

4. Meson decays: The bounds on meson decays into invisible final state (see e.g. Ref. [73] for a review) strongly limit the direct couplings of quarks, apart from the top, ${ }^{3}$ to the DM. In order to pass these constraints, one has to impose that $\Lambda_{D M}$ is larger than hundreds of $\mathrm{GeV}$ for low mass DM. Dimension6 operators with such a large scale of NP cannot guarantee the right DM relic abundance. Combining such bounds with the necessary small couplings $\lambda$ to avoid large Higgs invisible decay width, a DM particle coupling mainly to $u$-type and $d$-type quarks through $\mathcal{O}_{1, \ldots, 5}$ with mass $m_{D M}<m_{D} / 2$ and $m_{D M}<m_{B} / 2$, respectively, is then excluded.

5. Meson oscillations: Since no tree-level diagrams mediated by DM particles can be drawn, contributions to meson oscillations appear only at the loop level, as in the SM. The operators $\mathcal{O}_{1,2,3}$ give negligible contributions to the meson oscillation observables: the MFV ansatz ensures that the GIM mechanism also holds for these loop diagrams; an additional suppression $\propto 1 / \Lambda_{D M}^{4}$ makes NP contributions smaller than the corresponding SM ones. In contrast, the operators $\mathcal{O}_{4}$ and $\mathcal{O}_{5}$ have a LR chiral structure and the corresponding contributions to the meson oscillations are chiral enhanced. Following Ref. [49], the Wilson coefficients of the effective interactions $\left(\bar{q}_{R_{i}} q_{L_{j}}\right)\left(\bar{q}_{R_{i}} q_{L_{j}}\right)$, for $i>j$, depend on

$C_{R L}^{i j} \propto \frac{m_{q_{i}}^{2}}{32 \pi^{2} \Lambda_{D M}^{4}}\left(V_{t j}^{*} V_{t j}\right)^{2} F\left(\frac{m_{S_{h}}^{2}}{m_{S_{l}}^{2}}\right)$,

where $m_{S_{h}}\left(m_{S_{l}}\right)$ is the heaviest (lightest) of the $S$ components and $F(x)=((x+1) \log x) /(x-1)-2$ is the Inami-Lin function that vanishes in the limit $x \rightarrow 1$. For $\Lambda_{D M} \geqslant \mathrm{v}$, these NP contributions can be neglected while for $\Lambda_{D M} \ll v$, they can be large. Notice that for $K$ meson system, the Wilson coefficients are suppressed by $m_{s}^{2} / \Lambda_{D M}^{2}$ and no sizable effects can be seen in $\Delta M_{K}$ and $\epsilon_{K}$ (see Ref. [95] for a recent review for details on the meson oscillation observables). Let us also mention that for $B_{d}$ and $B_{s}$ systems, we expect new contributions in $\Delta M_{B_{d}}$ and $\Delta M_{B_{s}}$ only. Indeed, the absence of NP phases, in agreement with the MFV ansatz, prevents modifications on $S_{\psi K_{S}}$ and $S_{\psi \phi}$. In addition, let us stress that when the DM

\footnotetext{
3 In Ref. [70] it was argued that single top production at LHC with large missing transverse energy involving flavour violating interactions can give rise to novel signature for DM detection. Notice though that for the DM models studied here, the $t$ quarks only decay to $u$ and $c$ quarks and up to now LHC experiments can still not distinguish among light quarks jets.
}

components are very near in mass the Wilson coefficient get an extra suppression through the Inami-Lin function. This is typically the case when the DM relic abundance is driven by coannihilations.

For the models considered in this Letter, only the DM-quark interactions through $\mathcal{O}_{4,5}$ can thus contribute substantially to meson oscillations. In principle, the operator $\mathcal{O}_{4}$ can give rise to non-negligible contributions in $\bar{D}^{0}-D^{0}$ oscillations. The large theoretical uncertainties in the $D$ meson system prevents though to set relevant bounds on $\mathcal{O}_{4}$ mediated interactions. This is not the case in $B$ meson systems. In the presence of $\mathcal{O}_{5}$ interactions, we impose that NP contributions from (24) are at most equal to the theoretical uncertainties on $\Delta M_{B_{d}}$ and $\Delta M_{B_{s}}$, i.e. $\Delta C^{i j}=C_{R L}^{i j} / C_{S M}^{i j} \leqslant 0.1$.

Let us also mention that, the operators $\mathcal{O}_{4}$ and $\mathcal{O}_{5}$ induce also modifications of the quark mass terms, when contracting the two DM legs at loop to draw a tadpole diagram: these contributions can be safely neglected, providing relative corrections suppressed by loop factors and by $m_{D M}^{2} / \Lambda_{D M}^{2}$.

\subsection{Analytical insights}

As previously discussed, the Lorentz structure of the operators $\mathcal{O}_{1, \ldots, 5}$ can be categorised into two subgroups. The operators $\mathcal{O}_{1,2,3}$ could arise from vector boson exchange, while operators $\mathcal{O}_{4,5}$ would be associated to fermion or scalar exchange. At this point, it is instructive to analyse the scale of NP $\Lambda_{D M}$ that would a priori be necessary in order to give rise to the right relic abundance assuming a negligible contribution from Higgs portal. This is particularly relevant for DM masses below the Higgs resonance, where the bounds on the decay width of Higgs into invisible final state strongly constrains the DM-Higgs couplings $\lambda_{i}$ (see the colliders section in Section 4.1).

In the limit of low centre of mass velocity, $v \rightarrow 0$, the annihilation cross-sections associated to the processes $S_{i}^{*} S_{i} \rightarrow \bar{q}_{j} q_{j}$ for a fixed value of $i$ and $j$ become at leading order in $v$ :

$\left.\sigma v\right|_{\mathcal{O}_{1,2,3}} \simeq \frac{N_{c} c^{2} v^{2}}{48 \pi \Lambda_{D M}^{4}} \frac{m_{q}^{4}-5 m_{q}^{2} m_{D M}^{2}+4 m_{D M}^{4}}{m_{D M}^{2}\left(1-m_{q}^{2} / m_{D M}^{2}\right)^{1 / 2}}$

$\left.\sigma v\right|_{\mathcal{O}_{4,5}} \simeq \frac{N_{c} c^{\prime 2} m_{q}^{2}}{4 \pi \Lambda_{D M}^{4}}\left(1-m_{q}^{2} / m_{D M}^{2}\right)^{3 / 2}$

where $N_{c}$ is the number of colours of the final state quark, $c, c^{\prime}$ are a combination of $\mathcal{O}(1)$ coefficients and $m_{D M}$ and $m_{q}$ are the masses of $S_{i}$ and $q_{j}$ respectively. The first cross-section is p-wave suppressed while the second one corresponds to a s-wave driven process. Eq. (23) implies that for e.g. $m_{D M}=50 \mathrm{GeV}, \Lambda_{D M}$ should be $\sim 450 \mathrm{GeV}$ for $\mathcal{O}_{1}$ (five families of quarks are involved) and $\sim 200 \mathrm{GeV}$ for $\mathcal{O}_{5}$ (mainly $b$ quarks involved) in order to get the right relic abundance through annihilation only driven by the dimension- 6 operators. Let us emphasise though that given the velocity dependence of the $\sigma v$ 's above, it is clear that prospects for indirect detection, involving velocities $v \sim 10^{-3}$, will a priori be more constraining for $\mathcal{O}_{4,5}$ than $\mathcal{O}_{1,2,3}$. In practice, the coannihilations and Higgs portal interactions complicate the relic density analysis. The latter processes are fully taken into account in our numerical treatment of the DM models with the MicrOMEGAs code after having introduced the proper Feynman rules using the LanHEP package [96]. We have also used MicrOMEGAs's tools for the calculation of the cross-sections relevant for direct and indirect detection searches. 

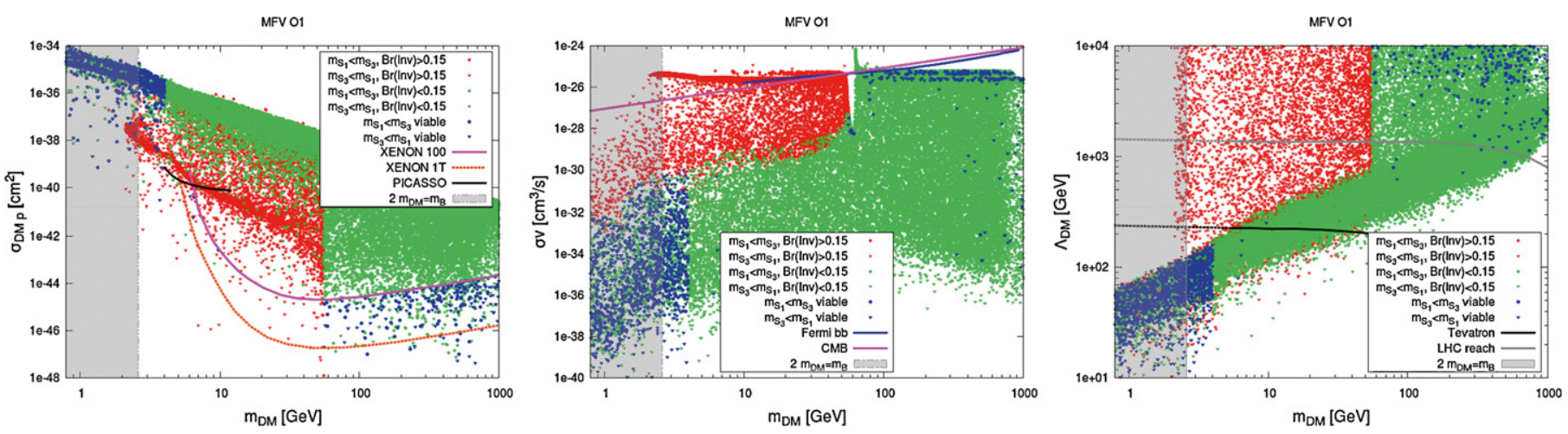

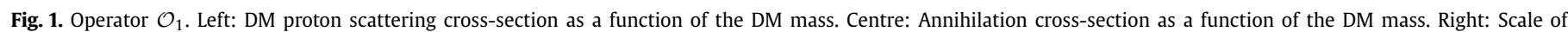

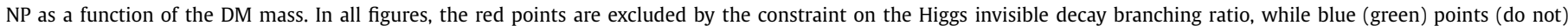

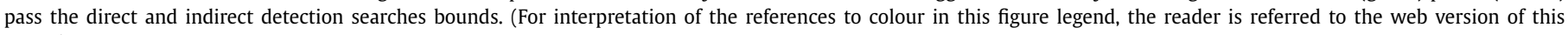
Letter.)

\subsection{Numerical analysis}

We have performed a random scan of the viable parameter space for DM within the following parameter ranges:

$$
\begin{aligned}
& 0.3<\left|a, a^{\prime}, b, b^{\prime}, c_{a}^{\alpha}\right|<1.3, \\
& 0.1 \mathrm{GeV}<m_{S_{3}}<1 \mathrm{TeV}, \\
& m_{D M}<\Lambda_{D M}<10 \mathrm{TeV}, \\
& 10^{-5}<|\lambda|<\pi
\end{aligned}
$$

considering real, positive and negative values for the parameters $a, a^{\prime}, b, b^{\prime}, c_{a}^{\alpha}$ and $\lambda$. Let us emphasise that once the value of $m_{S_{3}}$ is fixed, one can obtain larger or smaller values of $m_{S_{1}}$ and $m_{S_{2}}$ by scanning over $a, a^{\prime}, b, b^{\prime}$ and $\lambda$ parameters. As a result, in contrast to Ref. [49], we have not imposed in our analysis that $S_{3}$ is the lightest component of the $S$ triplet. The constraints from perturbativity, stability of the scalar potential, validity of the effective theory, and DM relic abundance are applied during the scan (see Section 4.1 for more details).

In Figs. 1 and 2, we present the results of the scans for the operators $\mathcal{O}_{1}$ and $\mathcal{O}_{4,5}$ in the planes $m_{D M}-\sigma_{D M p}, m_{D M}-\sigma v$ and $m_{D M}-\Lambda_{D M}$ superimposing the constraints from direct, indirect and colliders searches respectively. The corresponding plots for operators $\mathcal{O}_{2,3}$ are very similar to the ones for $\mathcal{O}_{1}$. The red points are excluded by constraints on the invisible decay branching ratio of $125 \mathrm{GeV}$ Higgs, while the blue (green) points (do not) pass the constraints from the combined PICASSO, Xenon 100, FERMI and $\mathrm{CMB}$ experiments.

Notice that in the $m_{D M}-\sigma v$ plots, we only show the constraint from FERMI on annihilation into $\bar{b} b$ at $100 \%$ as they are the most limiting in the model under study. The limit associated to CMB represented in the plots results from the analysis of Ref. [87]. The latter makes use of the recent SPT data assuming a value of the fraction of released energy in the IGM of 0.2 which should be representative of DM annihilating to quarks. ${ }^{4}$ In the legend of the plots, the "viable" reference should be interpreted as "passes direct, indirect detection and CMB constraints". In the $m_{D M}-\sigma_{D M p}$, we also present the reach of the future Xenon 1T experiment [97] that will test most of the large mass regime of $\mathcal{O}_{1, \ldots, 5}$ operators.

\footnotetext{
4 Notice that a similar analysis [88] shows the expected limits for each annihilation channels for WMAP7 and future Planck data release. It appears that the limits do not vary much going from annihilations into quarks to annihilation into gauge or Higgs boson. Also notice that Ref. [88] obtain a slightly less constraining bound for future Planck data. Here we stay a bit more conservative using the results of Ref. [87].
}

We will see that some blue points do not pass monojet searches constraints from Tevatron or are within the reach of LHC at $\sqrt{s}=$ $14 \mathrm{TeV}$. In Figs. 1 and 2, we represent with dotted lines the continuation of the limits from Ref. [94] to masses $m_{D M}<5 \mathrm{GeV}$. Notice however that in Ref. [94] slightly different assumptions were made on the coupling of DM to quarks compared to the present analysis. The lines in the $m_{D M}-\Lambda_{D M}$ associated to Tevatron and LHC constraints are thus mainly a guide for the eye. We thrust though that $\mathcal{O}_{1,2,3}$ low mass candidates are already excluded by such analysis (see Fig. 1), while for $\mathcal{O}_{4,5}$ only a few models are within the reach of LHC. A more detailed analysis of LHC prospects for detection of such a DM candidate is however beyond the scope of the present Letter.

\subsubsection{General features}

Several features appear in Figs. 1 and 2. Let us first concentrate on the $\sigma v-m_{D M}$ plots. Typically, when the correct relic abundance is achieved and it is driven by annihilation processes, the annihilation cross-section today reaches $\sim 3 \times 10^{-26} \mathrm{~cm}^{3} / \mathrm{s}$. In this $\sigma v$ range, we clearly see two preferred values of the annihilation cross-sections, especially in Fig. 2. The annihilation cross-section for $S_{1,2}$ DM today appears to be typically twice as large as the one for $S_{3}$.

This can be understood considering the fact that, in the MFV context, $S_{1}$ and $S_{2}$ are generally quite degenerate, see Section 2. In the latter case, the effective DM annihilation cross-section [74] driving the relic abundance goes as $\langle\sigma v\rangle \sim\left(\sigma v_{11}+\sigma v_{12}+\sigma v_{21}+\right.$ $\left.\sigma v_{22}\right) / 4$ where $\sigma v_{i j}$ refers to the cross-sections times velocity associated to $S_{i} S_{j}^{*} \rightarrow X$ annihilation and coannihilation processes ( $X$ is a SM final state) in the $v \rightarrow 0$ limit, assuming that the relic abundance is driven by s-wave dominated processes. When coannihilation processes are suppressed, i.e. $\sigma v_{i j i \neq j} \ll \sigma v_{i i}$ and $\sigma v_{i i}$ is identical for $i=1,2$, we get $\langle\sigma v\rangle=\sigma v_{11} / 2 \sim 3 \times 10^{-26} \mathrm{~cm}^{3} / \mathrm{s}$. This is what we observe in e.g. the upper plot of Fig. 2 when $\Lambda_{D M}$ is large and more especially when the interactions driven by $c_{2}^{\alpha}$ terms in Eqs. (21)-(22) are suppressed. Notice that we apply the detection searches constraints as if all the DM in the galaxies was made of only one species $S_{i}$. This is a rather conservative approach. If we had to consider that half of the DM is made of e.g. $S_{1}$ and the other half of $S_{2}$, some extra models with $m_{S_{1}}<m_{S_{3}}$ could be considered as viable.

Also notice that an important change in the number of relativistic degrees of freedom $g_{*}$ (from $\sim 100$ to $\sim 10$, see e.g. Ref. [98]) occurs in the early universe around ${ }^{5} T_{Q C D} \sim 150 \mathrm{MeV}$ due to the

\footnotetext{
5 A $T_{Q C D} \sim 150 \mathrm{MeV}$ has been taken into account in MicrOMEGAS code.
} 

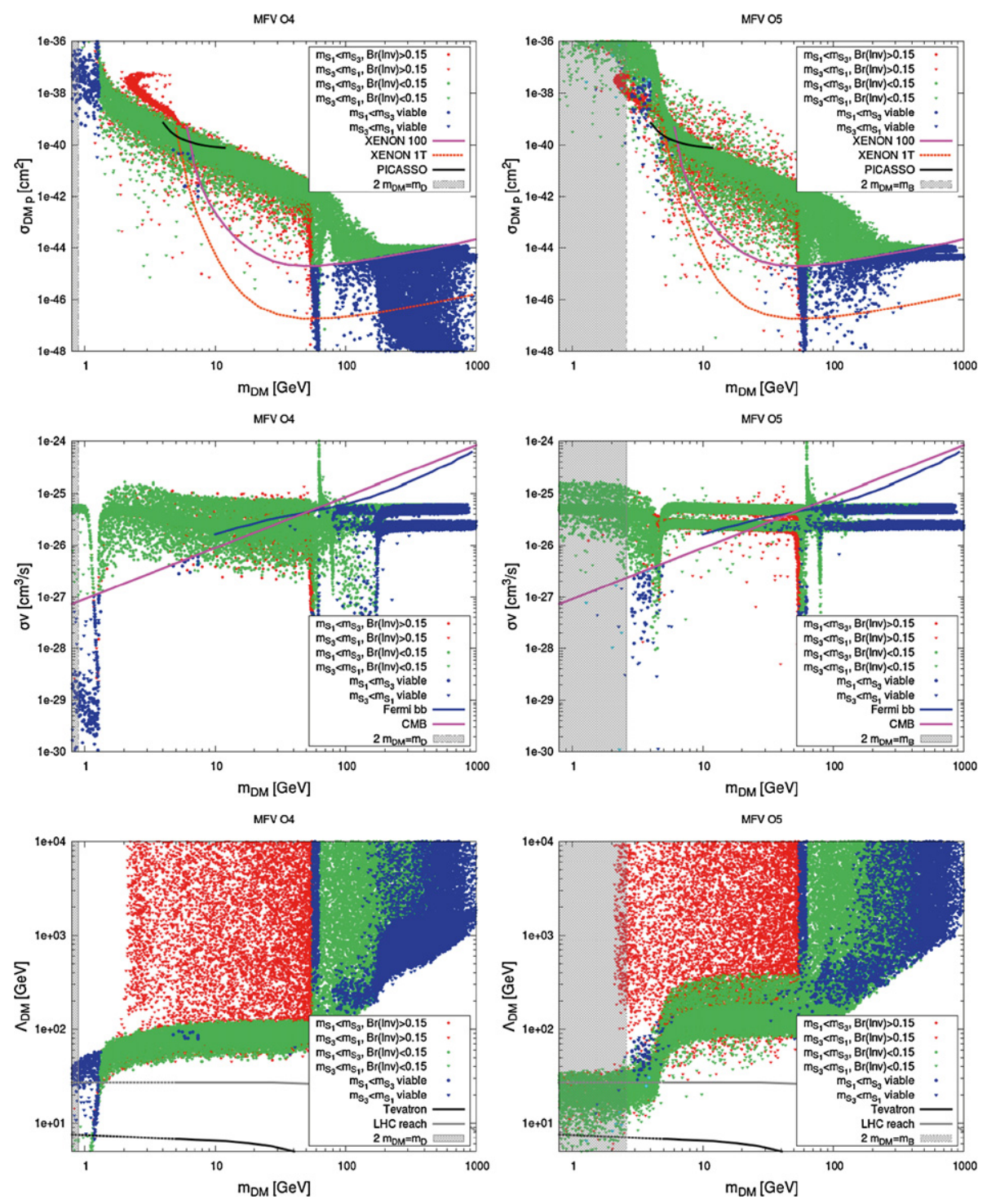

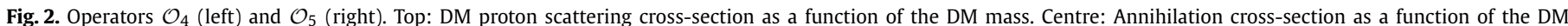

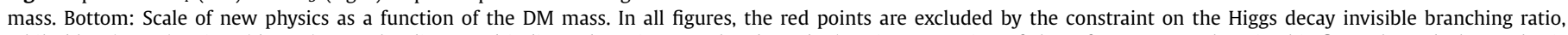

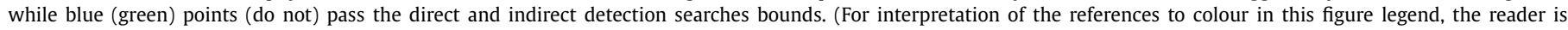
referred to the web version of this Letter.)

QCD phase transition. This implies larger values of the annihilation cross-section for $m_{D M} \sim T_{Q C D} x_{f}$.

The relative absence of points in the $m_{D M} \sim m_{h} / 2$ is due to resonant DM annihilation through Higgs exchange. This feature is also well visible in the $\Lambda_{D M}-m_{D M}$ and $\sigma_{D M}-m_{D M}$ plots. Another dip appears in all cases at $m_{D M} \sim m_{W}$ when the annihilation into gauge bosons through Higgs exchange become kinematically available. The other dips specific to the form of each operators are discussed in the following.

Considering now the $\Lambda_{D M}-m_{D M}$, we see that above the Higgs resonance the value of the $\Lambda_{D M}$ does not play an important role given that any value above $100 \mathrm{GeV}$ is allowed by all the constraints. Below the Higgs resonance, $\Lambda_{D M}$ should be typically smaller than $\mathcal{O}(100) \mathrm{GeV}$ to account for the DM relic abundance together with small contributions from the Higgs portal to evade a large correction to the Higgs width. Let us also remind that flavour observables, mainly meson decays into DM particles, impose that $\Lambda_{D M}$ is larger than hundreds of $\mathrm{GeV}$ for $m_{D M}$ in the $\mathrm{GeV}$ range [73]. More precisely, this constraint rules out $m_{D M}<m_{D} / 2$ and $m_{D M}<m_{B} / 2$ for the operators $\mathcal{O}_{4}$ and $\mathcal{O}_{1,5}$, respectively and the excluded regions are represented as (grey) shaded areas in Figs. 1 and 2. Moreover, the meson oscillation tends to constrain $\Delta C^{i j}<0.1$, see Section 4.1. This threatens the DM models of $\mathcal{O}_{5}$ with the lowest values of $\Lambda_{D M}$. The few points that are eventually excluded by this constraint are represented in light blue colours in the plots of the right column of Fig. 2.

The candidates that pass direct and indirect detection searches constraints (dark blue points) appear in two distinct mass ranges: below $10 \mathrm{GeV}$, where the direct detection bounds are weak, and above $m_{h} / 2$ as in the case of Higgs portal models [16]:

- For masses below $10 \mathrm{GeV}$, the scale of new physics is below $\sim 200 \mathrm{GeV}$ and the processes driving the relic abundance are typically related to the dimension- 6 operators $\mathcal{O}_{1, \ldots, 5}$.

- Around $60 \mathrm{GeV}$, near the Higgs resonance, the scale of new physics is typically above $1 \mathrm{TeV}$ and the coupling to the Higgs particle is small $(\lambda<0.01)$.

- Above $60 \mathrm{GeV}$, the scale of new physics is of several hundreds of $\mathrm{GeV}$ and the coupling to the Higgs particle is $\mathcal{O}(0.1-\pi)$. 


\subsubsection{Operators $\mathcal{O}_{1,2,3}$}

In Fig. 1, we present scatter plots associated to the operator $\mathcal{O}_{1}$. We have checked that equivalent results for $\mathcal{O}_{2,3}$ can be obtained (up to some $\mathcal{O}(1)$ factor in the $\Lambda_{D M}$ ). Given the p-wave suppression of the annihilation cross-section, see Eq. (25), it is the direct detection searches sector that provides the strongest constraints when $\mathcal{O}_{1}$ drives the DM relic abundance. In the low mass regime, they are limited by PICASSO's energy threshold at $m_{D M} \sim 4 \mathrm{GeV}$. Let us emphasise though that for scalar DM interacting through vector interactions with quarks, it has been shown [94] that monojet searches at Tevatron already provide very strong constraints on $\mathrm{GeV}$ range DM masses and rule out all the new potentially viable DM candidates with $m_{D M}<10 \mathrm{GeV}$, as shown in the plot on the right hand side of Fig. 1. For masses just above Higgs resonance, the presence of extra interactions through $\mathcal{O}_{1}$ allows for lower value of the $\sigma_{D M p}$ than in Higgs portal models and viable candidates that could be within the reach of future Xenon 1T experiments and in some rare cases will be tested by LHC at $\sqrt{s}=14 \mathrm{TeV}$.

\subsubsection{Operators $\mathcal{O}_{4,5}$}

In the case of the $\mathcal{O}_{4,5}$ operators, the results are shown in Fig. 2. This time the annihilation cross-section is an s-wave process so that the indirect detection searches can compete with direct detection bounds for $m_{D M}<10-20 \mathrm{GeV}$. In the $m_{D M}-\sigma v$ plane, in addition to the Higgs and gauge bosons resonances, some other dips appear at $m_{D M} \sim m_{c}, m_{t}$ in the $\mathcal{O}_{4}$ and $m_{D M} \sim m_{b}$ in the $\mathcal{O}_{5}$ case. They are due to the fact that the main DM annihilation channels into $S_{i}^{*} S_{i} \rightarrow \bar{c} c, \bar{t} t$ and $\bar{b} b$, respectively, are suppressed by factors of $\left(-m_{q}^{2}+m_{D M}^{2}\right)^{1 / 2}$ in the low velocity limit, see Eq. (25). Away from those dips and resonances, the points getting lower values of the annihilation cross-section have their relic abundance mainly driven by coannihilation processes.

As it can be seen in the bottom plots of Fig. 2, monojets searches [94] are not very limiting in the case of scalar type DMquarks interactions. The low mass regime appears to pass all constraints in contrast to the case of simple Higgs portal scenarios or to the case of Higgs portal $+\mathcal{O}_{1,2,3}$ considered in the previous section.

The particularities of operators $\mathcal{O}_{4}$ and $\mathcal{O}_{5}$ are the following:

4.3.3.1. Operator $\mathcal{O}_{4}:$ In the low mass range, viable DM candidates get an extra suppression of their relic abundance due to $S_{1} S_{2}^{*} \rightarrow u \bar{c}$ coannihilation process, driven by the interaction terms proportional to $c_{2}^{4}$ and $c_{4}^{4}$ in Eq. (21). For $m_{1,2}<m_{c}$, the coannihilation drives the relic density, while for $m_{1,2}>m_{c}$ it is a combination of coannihilations and $S_{2} S_{2}^{*} \rightarrow c \bar{c}$ that is relevant. Let us remind that in order to get a non-negligible contribution from coannihilations between two particles, their relative mass difference should be $\Delta m / m \sim 0.1$ in order to avoid exponential Boltzmann suppression. In the case of $\mathcal{O}_{4}$ no particular tuning in $m_{1,2}$ has to be invoked given that their degeneracy is granted thanks to the small $u$ and $c$ quarks Yukawa couplings in Eq. (9).

Also notice that in the case of $\mathcal{O}_{4}$, for $m_{D M}>m_{t}$ regime, one can obtain a very reduced value of $\sigma_{D M p}$ compared to minimal Higgs portal scenarios (see e.g. [16]). This typically happens in the case of negligible coupling to the Higgs so that $S_{i} S_{i}^{*} \rightarrow$ $W W, Z Z, \bar{t} t, h h$ through Higgs exchange are suppressed while $\mathcal{O}_{4}$ drives the DM relic abundance through $S_{i} S_{i}^{*} \rightarrow \bar{t} t$ for $m_{D M}>m_{t}$. This can be understood qualitatively by comparing the DM-proton scattering cross-sections for Higgs portal and $\mathcal{O}_{4}$ models. In the first case $\sigma_{D M p}^{h} \propto f_{p h}^{2} \lambda_{i}^{2} /\left(m_{h}^{4} m_{D M}^{2}\right) \mu^{2}$ [9] while in the latter case $\sigma_{D M p}^{\mathcal{O}_{4}} \propto f_{p 4}^{2} c^{2} /\left(\Lambda_{D M}^{4} m_{D M}^{2}\right) \mu^{2}$ [49] and $\mu$ is the DM-proton reduced mass. The nucleon form factors corresponds to $f_{p h} \simeq 0.4$ and $f_{p 4} \simeq 0.1$ in the Higgs portal and in the $\mathcal{O}_{4}$ case, respectively, using the MicrOMEGAs default parameters [76]. The differences between $f_{p h}$ and $f_{p 4}$ are principally due to the fact that $\mathcal{O}_{4}$ does not provide a coupling to the $s$ quark. For large DM masses, one can also relates the values of $\sigma v \propto g^{2} \lambda^{2} / m_{D M}^{2}$ and $\sigma v \propto c^{2} m_{t}^{2} / \Lambda_{D M}^{4}$ for a DM abundance driven by Higgs portal or $\mathcal{O}_{4}$ processes, respectively. Eventually we get for $m_{D M} \ll m_{t}$, a $\sigma_{D M p}^{\mathcal{O}_{4}}$ that is suppressed compared to $\sigma_{D M p}^{h}$ by a factor $\left(f_{p 4} / f_{p h}\right)^{2}$ and also by a factor $\sim m_{h}^{4} /\left(m_{t}^{2} m_{D M}^{2}\right)$.

4.3.3.2. Operator $\mathcal{O}_{5}$ : Below $10 \mathrm{GeV}$, viable candidates have their relic density mainly driven by coannihilations $S_{1} S_{3}^{*} \rightarrow \bar{b} d$ and $S_{2} S_{3}^{*} \rightarrow \bar{b}$ s. All these coannihilation channels are described by the flavour interactions proportional to $c_{2}^{5}$ and $c_{4}^{5}$ in Eq. (22). The $S_{1,2} S_{3}^{*}$ coannihilation processes require some tuning of the parameters. Eq. (9) gives rise to $m_{1}, m_{2} \sim\left|m_{A}\right|$ and $m_{3} \sim\left|m_{B}\right|$ so that, if $a^{(\prime)}, b^{(\prime)}=+1, m_{3} \simeq 2 m_{1,2}$. Allowing for different signs and the range of parameters in Eq. (26), we can get relative mass differences $\sim 0.1$ necessary for coannihilation.

From the above analysis appears that the coannihilation channels $S_{1} S_{2}^{*} \rightarrow u \bar{c}$ for $\mathcal{O}_{4}$ and $S_{1} S_{3}^{*} \rightarrow \bar{b} d$ and $S_{2} S_{3}^{*} \rightarrow \bar{b} s$ for $\mathcal{O}_{5}$ enable viable DM parameter space in the $\mathrm{GeV}$ range. This is due to the flavour interactions driven by $c_{2}^{\alpha}$ and $c_{4}^{\alpha}$ with $\alpha=4,5$ and this feature is absent in standard Higgs portal models. Notice that the $\mathrm{GeV}$ mass range will be tested in the next future by direct detection searches experiment, see e.g. Ref. [99] for SuperCDMS. Moreover, in the higher mass regime $m_{D M}>m_{t}, \mathcal{O}_{4}$ can give rise to rather suppressed DM-nucleon cross-sections that could even evade future Xenon 1T constraints.

\section{Conclusions}

Embedding the DM problematic within MFV context can guarantee DM stability [49]. In addition, flavour DM-fermions interactions, that naturally arise in this framework, give rise to a richer DM phenomenology than in the minimal Higgs portal scenarios (see e.g. [16] for a recent analysis). In this Letter, we focused on the MFV in the quark sector only with the Yukawas of the quarks being the only sources of flavour and $\mathrm{CP}$ violation. For definiteness, we have restricted our analysis to the case of scalar DM fields, neutral under the SM gauge symmetry, but transforming as a triplet under one of the $S U(3)$ composing the flavour symmetry group $G_{f}=S U(3)_{Q_{L}} \times S U(3)_{u_{R}} \times S U(3)_{d_{R}}$.

In this scenario, DM interacts with the SM fields through Higgs portal at renormalisable level and also to quarks through dimension- 6 operators. We have considered three vector operators and two scalar ones. We have performed a systematic analysis of the DM viable parameter space for each of these operators. We have obtained that DM candidates can pass direct and indirect DM detection searches constraints both below $10 \mathrm{GeV}$ and above $m_{h} / 2$.

Complementary constraints from colliders and flavour physics have been taken into account. In general the non-observation of meson decays into invisible final states exclude $m_{D M}<m_{D} / 2$ or $m_{D M}<m_{B} / 2$ mass ranges. In the case of dimension- 6 operators with DM-quarks vector like interactions, monojet searches at colliders rule out all the new potentially viable DM candidates with $m_{D M}<10 \mathrm{GeV}$. For what concerns scalar type dimension- 6 interactions, monojet searches are less limiting and allow for a viable $m_{D M}<10 \mathrm{GeV}$ parameter space that will probably evade future LHC monojet searches. In most cases, it also evades constraints from flavour violating processes such as meson oscillations. In the large mass regime, most of the viable parameter space will be within the reach of future direct detection searches experiments 
such as Xenon 1T, apart from the case of DM scalar-type coupling to $u$-type quarks that can evade the latter bound for $m_{D M}>m_{t}$.

To summarise, when the DM is embedded in the MFV framework, the DM stability is granted for certain representations of the DM fields under $G_{f}$. In this work, we systematically analysed the rich interplay between flavour and DM physics when considering vector and scalar type DM-quark interactions invariant under the original $G_{f}$ symmetry. Compared with minimal Higgs portal models, DM candidates with $m_{D M} \sim \mathrm{GeV}$ range are viable in these scenarios. Furthermore, DM particles with $m_{D M}>m_{t}$ could even be beyond the reach of future experiments such as Xenon 1T.

\section{Acknowledgements}

We thank Jean-Marie Frère, Belen Gavela, Michael Gustafsson, Alejandro Ibarra, Gino Isidori, Stefano Rigolin, Emmanuel Stamou and Michel Tytgat for useful discussions. LLH is supported through an "FWO-Vlaanderen" post-doctoral fellowship project number 1271513. LLH also recognises partial support from the Belgian Federal Science Policy Office through the Interuniversity Attraction Pole P7/37 and from the Strategic Research Program "High-Energy Physics" of the Vrije Universiteit Brussel. LM acknowledges partial support by European Union FP7 ITN INVISIBLES (Marie Curie Actions, PITN-GA-2011-289442) and the Juan de la Cierva programme (JCI-2011-09244).

\section{References}

[1] ATLAS Collaboration, G. Aad, et al., Phys. Lett. B 716 (2012) 1, arXiv:1207.7214.

[2] CMS Collaboration, S. Chatrchyan, et al., Phys. Lett. B 716 (2012) 30, arXiv: 1207.7235.

[3] B. Patt, F. Wilczek, hep-ph/0605188.

[4] Y.G. Kim, K.Y. Lee, Phys. Rev. D 75 (2007) 115012, hep-ph/0611069.

[5] J. March-Russell, S.M. West, D. Cumberbatch, D. Hooper, JHEP 0807 (2008) 058, arXiv:0801.3440.

[6] Y.G. Kim, K.Y. Lee, S. Shin, JHEP 0805 (2008) 100, arXiv:0803.2932.

[7] M. Ahlers, J. Jaeckel, J. Redondo, A. Ringwald, Phys. Rev. D 78 (2008) 075005, arXiv:0807.4143.

[8] J.L. Feng, H. Tu, H.-B. Yu, JCAP 0810 (2008) 043, arXiv:0808.2318.

[9] S. Andreas, T. Hambye, M.H. Tytgat, JCAP 0810 (2008) 034, arXiv:0808.0255.

[10] V. Barger, P. Langacker, M. McCaskey, M. Ramsey-Musolf, G. Shaughnessy, Phys. Rev. D 79 (2009) 015018, arXiv:0811.0393.

[11] M. Kadastik, K. Kannike, A. Racioppi, M. Raidal, Phys. Rev. Lett. 104 (2010) 201301, arXiv:0912.2729.

[12] S. Kanemura, S. Matsumoto, T. Nabeshima, N. Okada, Phys. Rev. D 82 (2010) 055026, arXiv:1005.5651.

[13] F. Piazza, M. Pospelov, Phys. Rev. D 82 (2010) 043533, arXiv:1003.2313.

[14] C. Arina, F.-X. Josse-Michaux, N. Sahu, Phys. Rev. D 82 (2010) 015005, arXiv: 1004.3953.

[15] I. Low, P. Schwaller, G. Shaughnessy, C.E. Wagner, Phys. Rev. D 85 (2012) 015009, arXiv: 1110.4405.

[16] A. Djouadi, O. Lebedev, Y. Mambrini, J. Quevillon, Phys. Lett. B 709 (2012) 65, arXiv:1112.3299.

[17] C. Englert, T. Plehn, D. Zerwas, P.M. Zerwas, Phys. Lett. B 703 (2011) 298, arXiv:1106.3097.

[18] J.F. Kamenik, C. Smith, Phys. Rev. D 85 (2012) 093017, arXiv:1201.4814.

[19] M. Gonderinger, H. Lim, M.J. Ramsey-Musolf, Phys. Rev. D 86 (2012) 043511, arXiv:1202.1316.

[20] O. Lebedev, Eur. Phys. J. C 72 (2012) 2058, arXiv:1203.0156.

[21] M. Farina, M. Kadastik, D. Pappadopulo, J. Pata, M. Raidal, et al., Nucl. Phys. B 853 (2011) 607, arXiv:1104.3572.

[22] M. Raidal, A. Strumia, Phys. Rev. D 84 (2011) 077701, arXiv:1108.4903.

[23] S. Baek, P. Ko, W.-I. Park, JHEP 1202 (2012) 047, arXiv:1112.1847.

[24] A. Djouadi, A. Falkowski, Y. Mambrini, J. Quevillon, arXiv:1205.3169.

[25] S. Andreas, C. Arina, T. Hambye, F.-S. Ling, M.H. Tytgat, Phys. Rev. D 82 (2010) 043522, arXiv:1003.2595.

[26] M. Gustafsson, S. Rydbeck, L. Lopez-Honorez, E. Lundstrom, Phys. Rev. D 86 (2012) 075019, arXiv:1206.6316.

[27] Y. Mambrini, M.H. Tytgat, G. Zaharijas, B. Zaldivar, JCAP 1211 (2012) 038, arXiv:1206.2352.

[28] Lei Wang, Xiao-Fang Han, Phys. Rev. D 87 (2013) 015015, arXiv:1209.0376.

[29] T. Hambye, PoS IDM 2010 (2011) 098, arXiv:1012.4587.

[30] E. Ma, Phys. Lett. B 671 (2009) 366, arXiv:0808.1729.
[31] M. Hirsch, S. Morisi, E. Peinado, J. Valle, Phys. Rev. D 82 (2010) 116003, arXiv: 1007.0871.

[32] N. Haba, Y. Kajiyama, S. Matsumoto, H. Okada, K. Yoshioka, Phys. Lett. B 695 (2011) 476, arXiv:1008.4777.

[33] D. Meloni, S. Morisi, E. Peinado, Phys. Lett. B 697 (2011) 339, arXiv:1011.1371.

[34] Y. Kajiyama, H. Okada, Nucl. Phys. B 848 (2011) 303, arXiv:1011.5753.

[35] M. Boucenna, M. Hirsch, S. Morisi, E. Peinado, M. Taoso, et al., JHEP 1105 (2011) 037, arXiv:1101.2874.

[36] A. Adulpravitchai, B. Batell, J. Pradler, Phys. Lett. B 700 (2011) 207, arXiv: 1103.3053.

[37] D. Meloni, S. Morisi, E. Peinado, Phys. Lett. B 703 (2011) 281, arXiv:1104.0178.

[38] R. de Adelhart Toorop, F. Bazzocchi, S. Morisi, Nucl. Phys. B 856 (2012) 670, arXiv:1104.5676.

[39] L. Lavoura, J. Phys. G 39 (2012) 025202, arXiv:1109.6854.

[40] K. Hashimoto, H. Okada, arXiv:1110.3640.

[41] M. Boucenna, S. Morisi, E. Peinado, Y. Shimizu, J. Valle, Phys. Rev. D 86 (2012) 073008, arXiv:1204.4733.

[42] L. Lavoura, S. Morisi, J. Valle, JHEP 1302 (2013) 118, arXiv:1205.3442.

[43] Y. BenTov, X.-G. He, A. Zee, arXiv:1208.1062.

[44] S. Bhattacharya, E. Ma, A. Natale, A. Rashed, arXiv:1302.6266.

[45] K. Hamaguchi, Y. Nomura, T. Yanagida, Phys. Rev. D 58 (1998) 103503, hepph/9805346.

[46] K. Hamaguchi, Y. Nomura, T. Yanagida, Phys. Rev. D 59 (1999) 063507, hepph/9809426.

[47] B. Batell, Phys. Rev. D 83 (2011) 035006, arXiv:1007.0045.

[48] M. Blennow, B. Dasgupta, E. Fernandez-Martínez, N. Rius, JHEP 1103 (2011) 014, arXiv:1009.3159.

[49] B. Batell, J. Pradler, M. Spannowsky, JHEP 1108 (2011) 038, arXiv:1105.1781.

[50] R.S. Chivukula, H. Georgi, Phys. Lett. B 188 (1987) 99.

[51] L.J. Hall, L. Randall, Phys. Rev. Lett. 65 (1990) 2939.

[52] G. D’Ambrosio, G. Giudice, G. Isidori, A. Strumia, Nucl. Phys. B 645 (2002) 155, hep-ph/0207036.

[53] V. Cirigliano, B. Grinstein, G. Isidori, M.B. Wise, Nucl. Phys. B 728 (2005) 121, hep-ph/0507001.

[54] S. Davidson, F. Palorini, Phys. Lett. B 642 (2006) 72, hep-ph/0607329.

[55] M. Gavela, T. Hambye, D. Hernandez, P. Hernandez, JHEP 0909 (2009) 038, arXiv:0906.1461.

[56] T. Feldmann, M. Jung, T. Mannel, Phys. Rev. D 80 (2009) 033003, arXiv:0906 1523.

[57] R. Alonso, M. Gavela, L. Merlo, S. Rigolin, JHEP 1107 (2011) 012, arXiv:1103. 2915.

[58] R. Alonso, G. Isidori, L. Merlo, L.A. Munoz, E. Nardi, JHEP 1106 (2011) 037, arXiv:1103.5461.

[59] R. Alonso, M. Gavela, D. Hernandez, L. Merlo, Phys. Lett. B 715 (2012) 194, arXiv:1206.3167.

[60] A.L. Fitzpatrick, G. Perez, L. Randall, Phys. Rev. Lett. 100 (2008) 171604, arXiv: 0710.1869

[61] A.L. Kagan, G. Perez, T. Volansky, J. Zupan, Phys. Rev. D 80 (2009) 076002, arXiv:0903.1794.

[62] Z. Lalak, S. Pokorski, G.G. Ross, JHEP 1008 (2010) 129, arXiv:1006.2375.

[63] B. Grinstein, M. Redi, G. Villadoro, JHEP 1011 (2010) 067, arXiv:1009.2049.

[64] A.J. Buras, M.V. Carlucci, L. Merlo, E. Stamou, JHEP 1203 (2012) 088, arXiv: 1112.4477.

[65] R. Alonso, M. Gavela, L. Merlo, S. Rigolin, J. Yepes, JHEP 1206 (2012) 076, arXiv: 1201.1511.

[66] R. Alonso, M. Gavela, L. Merlo, S. Rigolin, J. Yepes, arXiv:1212.3305.

[67] R. Alonso, M. Gavela, L. Merlo, S. Rigolin, J. Yepes, arXiv:1212.3307.

[68] J.M. Arnold, M. Pospelov, M. Trott, M.B. Wise, JHEP 1001 (2010) 073, arXiv: 0911.2225 .

[69] P. Agrawal, S. Blanchet, Z. Chacko, C. Kilic, Phys. Rev. D 86 (2012) 055002, arXiv:1109.3516.

[70] J.F. Kamenik, J. Zupan, Phys. Rev. D 84 (2011) 111502, arXiv:1107.0623.

[71] G. Isidori, Y. Nir, G. Perez, Ann. Rev. Nucl. Part. Sci. 60 (2010) 355, arXiv: 1002.0900

[72] E. Del Nobile, F. Sannino, Int. J. Mod. Phys. A 27 (2012) 1250065, arXiv:1102 3116.

[73] J.F. Kamenik, C. Smith, JHEP 1203 (2012) 090, arXiv:1111.6402.

[74] K. Griest, D. Seckel, Phys. Rev. D 43 (1991) 3191.

[75] G. Hinshaw, D. Larson, E. Komatsu, D. Spergel, C. Bennett, et al., arXiv:1212. 5226.

[76] G. Belanger, F. Boudjema, A. Pukhov, A. Semenov, Comput. Phys. Commun. 180 (2009) 747, arXiv:0803.2360.

[77] G. Belanger, F. Boudjema, P. Brun, A. Pukhov, S. Rosier-Lees, et al., Comput. Phys. Commun. 182 (2011) 842, arXiv:1004.1092.

[78] PICASSO Collaboration, S. Archambault, et al., Phys. Lett. B 711 (2012) 153 arXiv:1202.1240.

[79] XENON100 Collaboration, E. Aprile, et al., arXiv:1207.5988.

[80] Fermi-LAT Collaboration, M. Ackermann, et al., Phys. Rev. Lett. 107 (2011) 241302, arXiv:1108.3546. 
[81] Z. Hou, C. Reichardt, K. Story, B. Follin, R. Keisler, et al., arXiv:1212.6267.

[82] Planck Collaboration, astro-ph/0604069.

[83] N. Padmanabhan, D.P. Finkbeiner, Phys. Rev. D 72 (2005) 023508, astro-ph/ 0503486.

[84] A. Natarajan, D.J. Schwarz, Phys. Rev. D 80 (2009) 043529, arXiv:0903.4485.

[85] M. Cirelli, F. Iocco, P. Panci, JCAP 0910 (2009) 009, arXiv:0907.0719.

[86] D.P. Finkbeiner, S. Galli, T. Lin, T.R. Slatyer, Phys. Rev. D 85 (2012) 043522 arXiv:1109.6322.

[87] G. Giesen, J. Lesgourgues, B. Audren, Y. Ali-Haimoud, arXiv:1209.0247.

[88] J.M. Cline, P. Scott, arXiv:1301.5908.

[89] P.P. Giardino, K. Kannike, M. Raidal, A. Strumia, Phys. Lett. B 718 (2012) 469, arXiv:1207.1347.

[90] CMS Collaboration, S. Chatrchyan, et al., JHEP 1209 (2012) 094, arXiv:1206 5663.
[91] ATLAS Collaboration, G. Aad, et al., arXiv:1210.4491.

[92] M. Beltran, D. Hooper, E.W. Kolb, Z.C. Krusberg, Phys. Rev. D 80 (2009) 043509, arXiv:0808.3384.

[93] M. Beltran, D. Hooper, E.W. Kolb, Z.A. Krusberg, T.M. Tait, JHEP 1009 (2010) 037, arXiv:1002.4137.

[94] J. Goodman, M. Ibe, A. Rajaraman, W. Shepherd, T.M. Tait, et al., Phys. Rev. D 82 (2010) 116010, arXiv:1008.1783.

[95] G. Isidori, arXiv:1302.0661.

[96] A. Semenov, Comput. Phys. Commun. 180 (2009) 431, arXiv:0805.0555.

[97] M. Schumann, Slides of a talk given at the SPS Meeting 2012, ETH Zurich, 21.06.2012.

[98] E. Kolb, M. Turner, The Early Universe (Frontiers of Physics), Addison-Wesley Publishing Company, 1990.

[99] N. Mirabolfathi, Talk given at the Rencontres de Moriond 2013. 